\title{
SOME LIFTS OF DOUBLE VECTOR BUNDLES RELATED TO A PRODUCT PRESERVING GAUGE BUNDLE FUNCTOR ON VECTOR BUNDLES
}

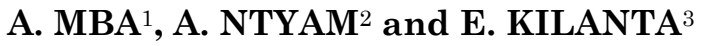 \\ ${ }^{1}$ Ecole Normale Supérieure \\ BP 47 Yaoundé \\ Cameroon \\ e-mail: alpmba@yahoo.fr \\ 2Department of Mathematics and Computer Science \\ Faculty of Science \\ The University of Ngaoundéré \\ P.O. Box 454, Ngaoundéré \\ Cameroon \\ e-mail: ntyam_achille@yahoo.fr \\ ${ }^{3}$ Department of Mathematics \\ Faculty of Science \\ The University of Ngaoundéré \\ P.O. Box 454, Ngaoundéré \\ Cameroon \\ e-mail: ekilanta@yahoo.com
}

\begin{abstract}
We present some lifts (associated to a product preserving bundle functor on vector bundles) of double vector bundles and linear sections on a double vector bundle.
\end{abstract}

2010 Mathematics Subject Classification: 58A32.

Keywords and phrases: double vector bundle, linear section, Weil bundle, gauge bundle functor, lift.

Received August 29, 2018; Revised September 22, 2018

(C) 2018 Scientific Advances Publishers 


\section{Introduction}

Weil functors (product preserving bundle functors on manifolds) were classified by [1], [10] and [5]. These functors were used by many authors (e.g., [2], [7], [8], [9]) to present some lifts of various geometric objects (smooth functions, tensor fields, linear connections on manifolds,...).

Product preserving gauge bundle functor on vector bundles (an example of bundle functors on local categories) were classified in [12]. Similarly to what is done for Weil functors some authors (e.g., [13], [14], [15]) present some lifts of some geometric objects related to product preserving gauge bundle functor on vector bundles.

The most fundamental example of a double vector bundle is the tangent bundle $T E$ of a vector bundle $(E, M, q)$. The two structures of vector bundles on $T E$ allow the development of some mathematical tools, namely linear connections, derivative endomorphisms, linear vector fields,....

In this paper, we present some lifts (associated to a product preserving gauge bundle functor on vector bundles) of double vector bundles and linear sections on double vector bundles.

\section{Product Preserving Gauge Bundle Functor on Vector Bundles}

\subsection{The Weil functor $T^{A}: \mathcal{M} f \rightarrow \mathcal{F M}$}

We write $\mathcal{M f}$ for the category of finite dimensional differential manifolds and mappings of class $C^{\infty}$; moreover, $\mathcal{F M}$ is the category of fibered manifolds and fibered manifolds morphisms.

Let us recall this construction of Weil functors based on [16]. For a Weil algebra $A=\mathbb{R} \cdot 1_{A} \oplus N$, i.e., a real commutative unital algebra where the ideal of nilpotent elements $N$ is a finite dimensional vector subspace of $A$, and any point $x$ of a differential manifold $M$, let $C_{x}^{\infty}(M, \mathbb{R})$ 
and $\operatorname{Hom}\left(C_{x}^{\infty}(M, \mathbb{R}), A\right)$ be the algebra of germs on $x$ of smooth functions and the set of algebra homomorphisms from $C_{x}^{\infty}(M, \mathbb{R})$ into $A$, respectively. If $\mathcal{E} n s$ denotes the category of sets and mappings, one defines a functor $T^{A}: \mathcal{M} f \rightarrow \mathcal{E} n s$ by:

$$
T^{A} M:=\bigcup_{x \in M} \operatorname{Hom}\left(C_{x}^{\infty}(M, \mathbb{R}), A\right) \text { and }\left(T^{A} f\right)_{x}\left(\varphi_{x}\right):=\varphi_{x} \circ f_{x}^{*}
$$

for a manifold $M$ and $f \in C^{\infty}\left(M, M^{\prime}\right)$, where $f_{x}^{*} \in \operatorname{Hom}\left(C_{f(x)}^{\infty}\right.$ $\left.\left(M^{\prime}, \mathbb{R}\right), C_{x}^{\infty}(M, \mathbb{R})\right)$ is the pull-back algebra homomorphism defined by $f^{*}\left(\operatorname{germ}_{f(x)}(h)\right)=\operatorname{germ}_{x}(h \circ f)$.

Now, let $q_{A, M}: T^{A} M \rightarrow M,\left(T^{A} M\right)_{x} \ni \varphi \mapsto x$; hence $\left(T^{A} M, M, q_{A, M}\right)$ is a well-defined fibered manifold. Indeed let $c=\left(U, u^{i}\right), 1 \leq i \leq m$ be a chart of $M$; then the map

$$
\begin{aligned}
\phi_{c}:\left(q_{A, M}\right)^{-1}(U) & \rightarrow U \times N^{m} \\
\varphi_{x} & \mapsto\left(x, \varphi_{x}\left(\operatorname{germ}_{x}\left(u^{i}-u^{i}(x)\right)\right),\right.
\end{aligned}
$$

is a local trivialization of $T^{A} M$. Given another manifold $M^{\prime}$ and a smooth map $f: M \rightarrow M^{\prime}, T^{A} f$ is a fibered map. Indeed for charts $c=(U, u, m), c^{\prime}=\left(W, w, m^{\prime}\right)$ of $M, M^{\prime}$ such that $f(U) \subset W, \phi_{c^{\prime}} \circ T^{A} f \circ \phi_{c}^{-1}$ is the map

$$
\begin{aligned}
U \times N^{m} & \rightarrow W \times N^{m^{\prime}} \\
\left(x, n_{i}\right) & \mapsto\left(f(x), n_{j}^{\prime}\right),
\end{aligned}
$$

where $n_{j}^{\prime}=\sum_{\alpha \in \mathbb{N}^{m} \backslash\{0\}} \frac{1}{\alpha !} D_{\alpha}\left(w^{j} \circ f \circ u^{-1}\right)(u(x)) n_{1}^{\alpha_{1}} \cdots n_{m}^{\alpha_{m}}, 1 \leq j \leq m^{\prime}$, with $D_{\alpha} F^{j}=\frac{\partial^{|\alpha|} F^{j}}{\left(\partial x^{1}\right)^{\alpha_{1}} \cdots\left(\partial x^{m}\right)^{\alpha_{m}}}$. 
$T^{A}: \mathcal{M f} \rightarrow \mathcal{F M}$ is a product preserving bundle functor (see [9]) called the Weil functor associated to $A$.

Let $c=(U, u)$ be a chart of $M$; in all the paper, we'll use fibered charts $\left(q_{A, M}^{-1}(U), u^{i, \alpha}\right), 1 \leq i \leq m, 0 \leq \alpha \leq \operatorname{dim} N$ of $T^{A} M$ associated to the fibered isomorphism $T^{A} u$ and defined by $u^{i, \alpha}=e_{\alpha}^{*} \circ T^{A}\left(u^{i}\right)$, where $\left(e_{\alpha}^{*}\right)$ is the dual basis of a fixed basis $\left(e_{\alpha}\right)_{0 \leq \alpha \leq \operatorname{dim} N}$ of $A$ such that $e_{0}=1$.

\subsection{Product preserving gauge bundle functor on $\mathcal{V B}$}

Let $F: \mathcal{V B} \rightarrow \mathcal{F M}$ be a covariant functor from the category $\mathcal{V B}$ (of vector bundles and vector bundles homomorphisms) into the category $\mathcal{F} \mathcal{M}$. Let $B_{\mathcal{V B}}: \mathcal{V B} \rightarrow \mathcal{M} f$ and $B_{\mathcal{F M}}: \mathcal{F M} \rightarrow \mathcal{M} f$ be the respective base functors.

Definition 2.1. $F$ is a gauge bundle functor on $\mathcal{V B}$ when the following conditions are satisfied:

- (Prolongation) $\mathcal{B}_{\mathcal{F M}} \circ F=B_{\mathcal{V B}}$, i.e., $F$ transforms a vector bundle $E \stackrel{q}{\rightarrow} M$ in a fibered manifold $F E \stackrel{p_{E}}{\rightarrow} M$ and a vector bundle morphism $E \stackrel{f}{\rightarrow} G$ over $M \stackrel{\bar{f}}{\rightarrow} N$ in a fibered map $F E \stackrel{F f}{\rightarrow} F G$ over $\bar{f}$.

- (Localization) For any vector bundle $E \stackrel{q}{\rightarrow} M$ and any inclusion of an open vector subbundle $i: q^{-1}(U) \hookrightarrow E$, the fibered map $F q^{-1}(U) \rightarrow$ $p_{E}^{-1}(U)$ over $i d_{U}$ induced by $F i$ is an isomorphism then the map $F i$ can be identified to the inclusion $p_{E}^{-1}(U) \hookrightarrow F E$. 
Given two gauge bundle functors $F_{1}, F_{2}$ on $\mathcal{V B}$, by a natural transformation $\tau: F_{1} \rightarrow F_{2}$ we shall mean a system of base preserving fibered maps $\tau_{E}: F_{1} E \rightarrow F_{2} E$ for every vector ${ }_{M}$ bundle $E$ satisfying $F_{2} f \circ \tau_{E}=\tau_{G} \circ F_{1} f$ for every vector bundle morphism $f: E \rightarrow G$.

A gauge bundle functor $F$ on $\mathcal{V B}$ is product preserving if for any product projections $E_{1} \stackrel{p r_{1}}{\longleftarrow} E_{1} \times E_{2} \stackrel{p r_{2}}{\longrightarrow} E_{2}$ in the category $\mathcal{V B}, F E_{1}$ $\stackrel{F p r_{1}}{\longleftarrow} F\left(E_{1} \times E_{2}\right) \stackrel{F p r_{2}}{\longrightarrow} F E_{2}$ are product projections in the category $\mathcal{F M}$. In other words, the map $\left(F p r_{1}, F p r_{2}\right): F\left(E_{1} \times E_{2}\right) \rightarrow F\left(E_{1}\right) \times F\left(E_{2}\right)$ is a fibered isomorphism over $i d_{M_{1} \times M_{2}}$.

Example 2.1. Let $A=\mathbb{R} \cdot 1_{A} \oplus N$ be a Weil algebra.

(a) Each Weil functor $T^{A}$ induces a product preserving gauge bundle functor $T^{A}: \mathcal{V B} \rightarrow \mathcal{F M}$ in a natural way.

(b) The gauge bundle functor $T^{A, V}: \mathcal{V B} \rightarrow \mathcal{F M}$ : Let $V$ be a $A$-module such that $\operatorname{dim}_{\mathbb{R}}(V)<\infty$. For a vector bundle $(E, M, q)$ and $x \in M$, let $T_{x}^{A, V} E=\left\{\left(\varphi_{x}, \psi_{x}\right) / \varphi_{x} \in \operatorname{Hom}\left(C_{x}^{\infty}(M, \mathbb{R}), A\right)\right.$ and $\left.\psi_{x} \in \operatorname{Hom}_{\varphi_{x}}\left(C_{x}^{\infty, f, l}(E), V\right)\right\}$, where $\operatorname{Hom}\left(C_{x}^{\infty}(M, \mathbb{R}), A\right)$ is the set of algebra homomorphisms $\varphi_{x}$ from the algebra $C_{x}^{\infty}(M, \mathbb{R})=\left\{\operatorname{germ}_{x}(g) / g \in C^{\infty}(M, \mathbb{R})\right\}$ into $A$ and $\operatorname{Hom}_{\varphi_{x}}\left(C_{x}^{\infty, f, l}(E), V\right)$ is the set of module homomorphisms $\psi_{x}$ over $\varphi_{x}$ from the $C_{x}^{\infty}(M, \mathbb{R})$-module $C_{x}^{\infty, f, l}(E, \mathbb{R})=\left\{\operatorname{germ}_{x}(h) / h: E \rightarrow \mathbb{R}\right.$ is fiberwise linear\} into $V$. Let $T^{A, V} E=\bigcup_{x \in M} T_{x}^{A, V} E$ and $p_{E}^{A, V}: T^{A, V} E \rightarrow M$, $T_{x}^{A, V} E \ni(\varphi, \psi) \mapsto x .\left(T^{A, V} E, M, p_{E}^{A, V}\right) \quad$ is $\quad$ a $\quad$ well-defined fibered 
manifold. Indeed let $c=\left(q^{-1}(U), x^{i}=u^{i} \circ q, y^{j}\right), 1 \leq i \leq m, 1 \leq j \leq n$ be a fibered chart of $E$; then the map

$$
\begin{aligned}
\phi_{c}:\left(p_{E}^{A, V}\right)^{-1}(U) & \rightarrow U \times N^{m} \times V^{n} \\
\left(\varphi_{x}, \psi_{x}\right) & \mapsto\left(x, \varphi_{x}\left(\operatorname{germ}_{x}\left(u^{i}-u^{i}(x)\right)\right), \psi_{x}\left(\operatorname{germ}_{x}\left(y^{j}\right)\right)\right),
\end{aligned}
$$

is a local trivialization for a bundle structure on $T^{A, V} E$. Given another vector bundle $\left(G, M^{\prime}, q^{\prime}\right)$ and a vector bundle homomorphism $f: E \rightarrow G$ over $\bar{f}: M \rightarrow M^{\prime}$, let

$$
\begin{aligned}
T^{A, V} f: T^{A, V} E & \rightarrow T^{A, V_{G}} \\
\left(\varphi_{x}, \varphi_{x}\right) & \mapsto\left(\varphi_{x} \circ \bar{f}_{x}^{*}, \varphi_{x} \circ f_{x}^{*}\right),
\end{aligned}
$$

where $\quad \bar{f}_{x}^{*}: C_{\bar{f}(x)}^{\infty}(N) \rightarrow C_{x}^{\infty}(M) \quad$ and $\quad f_{x}^{*}: C_{\bar{f}(x)}^{\infty, f, l}(G) \rightarrow C_{x}^{\infty, f, l}(E) \quad$ are given by the pull-back with respect to $\bar{f}$ and $f$. Then $T^{A, V} f$ is a fibered map over $\bar{f} . T^{A, V}: \mathcal{V B} \rightarrow \mathcal{F M}$ is a product preserving gauge bundle functor (see [12]).

Remark 2.1. Let $F: \mathcal{V B} \rightarrow \mathcal{F M}$ be a product preserving gauge bundle functor.

(a) $F$ associates the pair $\left(A^{F}, V^{F}\right)$, where $A^{F}=F\left(i d_{\mathbb{R}}: \mathbb{R} \rightarrow \mathbb{R}\right)$ is a Weil algebra and $V^{F}=F(\mathbb{R} \rightarrow p t)$ is a $A^{F}$-module such that $\operatorname{dim}_{\mathbb{R}}\left(V^{F}\right)<\infty$.

(b) There is a natural isomorphism $\Theta: F \rightarrow T^{A^{F}, V^{F}}$ defined on the vector bundle $(E, M, q)$ as follows : For $\xi \in F_{x} E, \Theta_{E}(\xi)=\left(\varphi_{x}, \psi_{x}\right)$ with $\varphi_{x}\left(\operatorname{germ}_{x} f\right)=F(f \circ q)(\xi)$ and $\psi_{x}\left(\operatorname{germ}_{x}(h)\right)=F(h)(\xi)$. In particular, the product preserving gauge bundle functor $T^{A}: \mathcal{V B} \rightarrow \mathcal{F M}$ is equivalent to $T^{A, A}$. 
(c) For two vector bundles $E_{1}, E_{2}$ with the same base $M$ and $p_{i}: E_{1}$ $\oplus_{M} E_{2} \rightarrow E_{i}, i=1,2$ the projections, the map $\left(F p_{1}, F p_{2}\right): F\left(E_{1} \oplus_{M}\right.$ $\left.E_{2}\right) \rightarrow F E_{1} \times F E_{2}$ induces a vector bundle isomorphism $F\left(E_{1} \oplus_{M} E_{2}\right)$ $\rightarrow F E_{1} \oplus_{F M} F E_{2}$ in a natural way.

(d) For a vector bundle $(E, M, q),(F E, F M, F q)$ is also a vector bundle where $F M=T^{A^{F}} M$; if $f: E \rightarrow G$ is a morphism of vector bundles over $\bar{f}: M \rightarrow M^{\prime}$, then $F f: F E \rightarrow F G$ is also a morphism of vector bundles over $F \bar{f}: F M \rightarrow F M^{\prime}$. The addition, the scalar multiplication and the zero section of $F E \rightarrow F M \rightarrow F M$ are respectively, given by

$$
\begin{array}{rlrl}
F E \oplus_{F M} F E & \rightarrow F E & \mathbb{R} \times F E & \rightarrow F E \\
(\tilde{u}, \widetilde{v}) & \mapsto F\left(a d^{E}\right)(\tilde{u}, \widetilde{v})^{\prime} & (\lambda, \tilde{u}) & \mapsto F\left(m_{\lambda}^{E}\right)(\widetilde{u}), \\
& & \text { and } F 0_{E}: F M \rightarrow F E,
\end{array}
$$

with $a d^{E}, m^{E}$, and $0_{E}$ the addition, scalar multiplication, and zero section of $E$, respectively.

\section{Lifts of Double Vector Bundles}

\subsection{Double vector bundles}

Definition 3.1. A double vector bundle structure is a system $(D, A, B, M)$ of four vector bundles structures

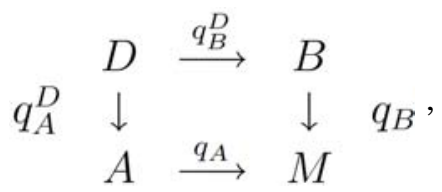

where $D$ is a vector bundle on bases $A$ and $B$, which are themselves vector bundles on $M$, such that each of the four structure maps of each 
vector bundle structure on $D$ (namely, the bundle projection, addition, scalar multiplication and the zero section) is vector bundle morphism with respect to other structure.

Remark 3.1. Let $(D, A, B, M)$ be a double vector bundle. The last part of the definition means that:

(1) $q_{B}^{D}$ and $q_{A}^{D}$ are morphisms of vector bundles over $q_{A}$ and $q_{B}$, respectively; in particular $q_{A} \circ q_{A}^{D}=q_{B} \circ q_{B}^{D}: D \rightarrow M$.

(2) The zero sections $0_{A}^{D}: A \rightarrow D, 0_{B}^{D}: B \rightarrow D$ are morphisms of vector bundles over the zero sections $0_{M}^{A}: M \rightarrow A, 0_{M}^{B}: M \rightarrow B$, respectively.

(3) Since the sum $D \oplus_{B} D$ is a subbundle of the vector bundle

$$
\left(q_{A}^{D} \times q_{A}^{D}\right)^{-1}\left(A \oplus_{M} A\right) \mapsto A \oplus_{M} A
$$

by (1) and (2), the addition $a d_{B}^{D}: D \oplus_{B} D \rightarrow D$ is a morphism of vector bundles over the addition $a d_{M}^{A}: A \oplus_{M} A \rightarrow A$. Similarly, the addition $a d_{A}^{D}: D \oplus_{A} D \rightarrow D$ is a morphism of vector bundles over the addition $a d_{M}^{B}: B \oplus_{M} B \rightarrow M$.

(4) The scalar multiplication of $D \rightarrow B, m_{B}^{D}: \mathbb{R} \times D \rightarrow D$ is a morphism of vector bundles over the scalar multiplication $m_{M}^{A}: \mathbb{R} \times A \rightarrow A$. Similarly, of $D \rightarrow A, m_{A}^{D}: \mathbb{R} \times D \rightarrow D$ is a morphism of vector bundles over the scalar multiplication $m_{M}^{B}: \mathbb{R} \times B \rightarrow B$.

Definition 3.2. Let $(D, A, B, M)$ be a double vector bundle. The bundle $D \stackrel{q_{B}^{D}}{\rightarrow} B$ is called the horizontal bundle structure on $D$ and $D \stackrel{q_{A}^{D}}{\rightarrow} A$ is called the vertical bundle structure on $D$. 
Example 3.1. (a) For a differential manifold $M$, let us consider trivial vector bundles $A=M \times \mathbb{R}^{n}, B=M \times \mathbb{R}^{p}, D=M \times \mathbb{R}^{n} \times \mathbb{R}^{p} \times \mathbb{R}^{q}$ over $A$ and $B$; then $(D, A, B, M)$ is a double vector bundle.

(b) The tangent bundle of a vector bundle $(E, M, p)$

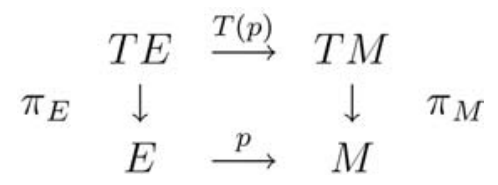

is a double vector bundle.

Definition 3.3. A morphism of double vector bundles

$$
\left(\varphi, \varphi_{A}, \varphi_{B}, f\right):(D, A, B, M) \rightarrow\left(D^{\prime}, A^{\prime}, B^{\prime}, M^{\prime}\right)
$$

consists of morphisms of vectors bundles

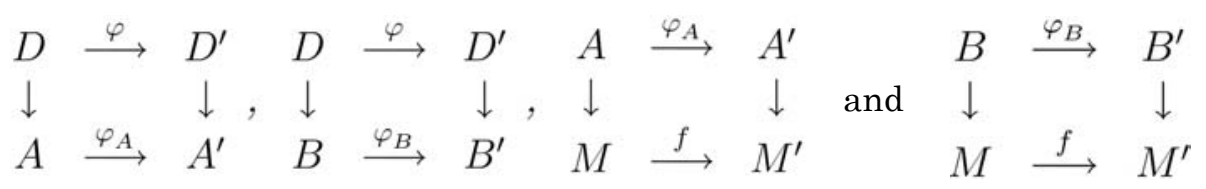

If $M=M^{\prime}$ and $f=i d_{M}, \varphi$ is called a morphism over $M$; if further $A=A^{\prime}$ and $\varphi_{A}=i d_{A}, \varphi$ is said over $A$. If $A=A^{\prime}, B=B^{\prime}$ and both $\varphi_{A}, \varphi_{B}$ are identities, we say that $\varphi$ preserves the side bundles.

\subsection{Double vector bundles as homogeneous structures}

Let us recall the following notions according to [3].

A smooth action of the multiplicative monoid $\left(\mathbb{R}_{+}, \cdot\right)$ on a smooth manifold $F$, is a smooth map $h: \mathbb{R}_{+} \times F \rightarrow F,(t, x) \mapsto h(t, x)=h_{t}(x)$ such that $h(1, x)=x$ and $h_{t} \circ h_{s}=h_{t s}$. 
Given a smooth action $h: \mathbb{R}_{+} \times F \rightarrow F$, let $M=h_{0}(F)$ the set of fixed points of the projection $h_{0}$ and the smooth map

$$
\mathcal{V}: F \rightarrow T F,\left.x \mapsto \frac{d}{d t}\right|_{t=0} h(t, x) \in T_{h_{0}(x)} F
$$

It is clear that $M \subset \mathcal{V}^{-1}(0)$, the inverse image of the set of zeros of $T F$; if $M=\mathcal{V}^{-1}(0) h$ is called a homogeneous structure on $F$. In this case, there is a structure of vector bundle $E \rightarrow M$ on $E=\mathcal{V}(F)$ and $\mathcal{V}: F \rightarrow E$ is a diffeomorphism. The vector bundle structure $F \stackrel{h_{0}}{\rightarrow} M$ carried by this diffeomorphism is the unique vector bundle structure on $F$ whose homotheties coincide with $h$. Conversely homotheties of a vector bundle $F$ associate a homogeneous structure $h: \mathbb{R}_{+} \times F \rightarrow F,(t, x) \mapsto t \cdot x$. This implies that vector bundles correspond with homogeneous structures.

The Euler vector field of a vector bundle $F \rightarrow M$ is the smooth vector field $\Delta_{F}$ on $F$ given by $\Delta_{F}(x)=\left.\frac{d}{d t}\right|_{t=1} t \cdot x$. The global flow $F l^{\Delta_{E}}: \mathbb{R} \times F \rightarrow F$ of $\Delta_{F}$ is given by $F l^{\Delta_{E}}(t, x)=e^{t} \cdot x=h\left(e^{t}, x\right)$.

Two homogeneous structures $h_{1}, h_{2}: \mathbb{R}_{+} \times F \rightarrow F$ are called commuting homogeneous structures if $h_{t}^{1} \circ h_{s}^{2}=h_{s}^{2} \circ h_{t}^{1}$ for all $(s, t) \in \mathbb{R}_{+} \times \mathbb{R}_{+}$i.e., $\left[\Delta^{1}, \Delta^{2}\right]=0$, since $h_{1}, h_{2}$ come from global flows of $\Delta^{1}, \Delta^{2}$. If one denotes $E^{i}=h_{0}^{i}(F), i=1,2$ the corresponding bases and $M=E^{1} \cap E^{2}$, the system

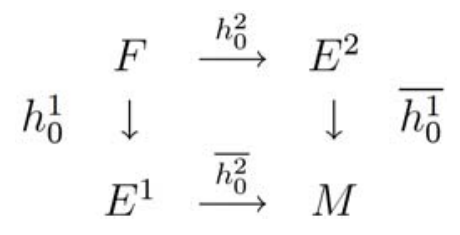


is a double vector bundle structure. Conversely, the homotheties of a double vector bundle

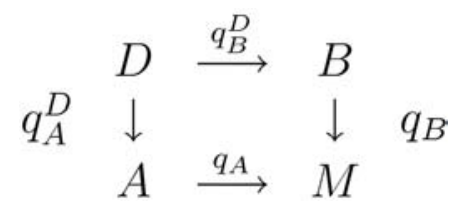

commute. Indeed, the scalar multiplication of $D \rightarrow B, m_{B}^{D}: \mathbb{R} \times D \rightarrow D$ is a morphism of vector bundles over the scalar multiplication $m_{M}^{A}: \mathbb{R}$ $\times A \rightarrow A$, hence for $(s, a) \in \mathbb{R} \times A$, the induced map $\{s\} \times D_{a} \rightarrow D_{s \cdot a}$, $(s, d) \mapsto s_{\dot{B}} d$ is linear; in particular for all $t \in \mathbb{R}, s_{\dot{B}}(t \dot{\dot{A}} d)=t_{\dot{A}}\left(s_{\dot{B}} d\right)$. This shows that a double vector bundle can be equivalently defined as a smooth manifold equipped with two vector bundle structures whose Euler vector fields $\Delta^{1}, \Delta^{2}$ commute.

\subsection{Natural transformations $Q(a): F \rightarrow F$}

Let $F: \mathcal{V B} \rightarrow \mathcal{F M}$ be a product preserving gauge bundle functor.

For a vector bundle $(E, M, q)$, the scalar multiplication $m_{M}^{E}: \mathbb{R} \times E \rightarrow E$, is a vector bundle morphism over the projection $p r_{2}: \mathbb{R} \times M \rightarrow M$, hence for any $a \in A^{F}$, there is a natural transformation $Q(a): F \rightarrow F$ given by

$$
Q(a)_{E}=F m_{M}^{E}(a, \cdot): F E \rightarrow F E .
$$

$Q(a)$ is entirely determined by the algebra homomorphism $Q(a)_{\mathbb{R}} \stackrel{i d_{\mathbb{R}}}{\rightarrow} \mathbb{R}=$ $i d_{A^{F}}$ and the module homomorphism $Q(a)_{\mathbb{R} \rightarrow p t}: V^{F} \rightarrow V^{F}, v \mapsto a \cdot v$, over $i d_{A^{F}}$ (Theorem 3.5 [12]). The following result is clear. 
Proposition 3.1. We have

(1) $Q\left(1_{A}\right)=i d_{T^{A}}$,

(2) $Q(a+b)=Q(a)+Q(b)$,

(3) $Q(\lambda \cdot a)=\lambda \cdot Q(a)$

(4) $Q(a b)=Q(a) \circ Q(b)$,

for all $\lambda \in \mathbb{R}$ and $(a, b) \in A^{2}$.

\subsection{Lifts of double vector bundles and linear sections}

Let $F: \mathcal{V B} \rightarrow \mathcal{F M}$ be a product preserving gauge bundle functor and $(D, A, B, M)$ a double vector bundle.

Theorem 3.2. The system (FD, FA, FB, FM) is also a double vector bundle.

Proof. (1) $F q_{B}^{D}$ and $F q_{A}^{D}$ are morphisms of vector bundles over $F q_{A}$ and $F q_{B}$, respectively by Remark 3.1(d).

(2) The zero sections $F 0_{A}^{D}: F A \rightarrow F D, F 0_{B}^{D}: F B \rightarrow F D$ are morphisms of vector bundles over the zero sections $F 0_{M}^{A}: F M \rightarrow F A$, $F 0_{M}^{B}: F M \rightarrow F B$ by the same remark.

(3) The additions $\operatorname{Fad}_{B}^{D}: F D \oplus_{F B} F D \rightarrow F D, F_{A d}^{D}: F D \oplus_{F A} F D \rightarrow F D$ are morphisms of vector bundles over the addition $\operatorname{ad}_{M}^{A}: A \oplus_{M} A \rightarrow A$, $a d_{M}^{B}: B \oplus_{M} B \rightarrow M$, respectively.

(4) The scalar multiplications of $F D \rightarrow F B, F D \rightarrow F A$

$$
\begin{aligned}
m_{F B}^{F D}: \mathbb{R} \times F D & \rightarrow F D & m_{F A}^{F D}: \mathbb{R} \times F D & \rightarrow F D \\
(\lambda, \tilde{d}) & \mapsto F\left(m_{B \lambda}^{D}\right)(\tilde{d}), & (\lambda, \tilde{d}) & \mapsto F\left(m_{A \lambda}^{D}\right)(\tilde{d})
\end{aligned}
$$


are morphisms of vector bundles over the scalar multiplications

$$
\begin{aligned}
m_{F M}^{F A}: \mathbb{R} \times F A & \rightarrow F A & m_{F M}^{F B}: \mathbb{R} \times F B & \rightarrow F B \\
(\lambda, \tilde{a}) & \mapsto F\left(m_{M \lambda}^{A}\right)(\widetilde{a}), & (\lambda, \tilde{a}) & \mapsto F\left(m_{M \lambda}^{B}\right)(\widetilde{a}),
\end{aligned}
$$

respectively, since by for each $\lambda \in \mathbb{R}$, the partial maps $m_{B \lambda}^{D}$ and $m_{A \lambda}^{D}$ are morphisms of vector bundles over $m_{M \lambda}^{A}$ and $m_{M \lambda}^{B}$, respectively.

Remark 3.2. Let us denote $h_{s}^{A}, h_{t}^{B}(s, t) \in \mathbb{R}^{*} \times \mathbb{R}^{*}$ the homotheties of $F D \rightarrow F A, F D \rightarrow F B$, respectively. These are commuting vector bundle morphisms, hence $F h_{s}^{A}, F h_{t}^{B}(s, t) \in \mathbb{R}^{*} \times \mathbb{R}^{*}$ commute and since they are exactly homotheties of $F D \rightarrow F A, F D \rightarrow F B$,

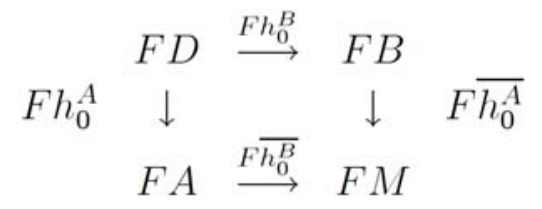

is a double vector bundle.

Let $(D, A, B, M)$ be a double vector bundle. We fix one of structures of vector bundles on $D, q_{B}^{D}: D \rightarrow B$ for instance.

Definition 3.4. A smooth section $\sigma \in \Gamma\left(q_{B}^{D}\right)$ is said linear if it is a morphism of vector bundles

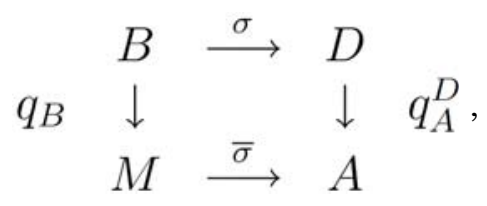

i.e., $h_{t}^{A} \circ \sigma=\sigma \circ h_{t}$, for all $t \in \mathbb{R}_{+}$, where $h, h^{A}$ are homotheties of $B \rightarrow M, D \rightarrow A$, respectively (Theorem 2.4 [3]). 
Example 3.2. Linear sections of the double vector bundle (TE, TM, E, M) associated to the vector bundle structure $\pi_{E}: T E \rightarrow E$ are called linear vector fields on $E$. Linear vector fields were studied in [11] and classified in [13]. Some properties of lifts of such vector fields were studied in [14] and [15].

Definition 3.5. For a smooth linear section $\sigma: B \rightarrow D$ over $\bar{\sigma}: M \rightarrow A$ its a-lift $\left(a \in A^{F}\right)$ related to $F$ is given by $\sigma^{(a)}=Q(a)_{D} \circ F_{\sigma}: F B \rightarrow F D$, where $Q(a): F \rightarrow F$ is the natural transformation (3.1).

$$
\begin{aligned}
& \sigma^{(a)} \in \Gamma\left(F q_{B}^{D}\right) \text { is linear over } \bar{\sigma}^{(a)} \in \Gamma\left(F q_{A}\right) \text { since } \\
& \begin{aligned}
F q_{A}^{D} \circ \sigma^{(a)} & =F q_{A}^{D} \circ Q(a)_{D} \circ F \sigma \\
& =Q(a)_{A} \circ F q_{A}^{D} \circ F \sigma \\
& =Q(a)_{A} \circ F\left(\bar{\sigma} \circ q_{B}\right) \\
& =\bar{\sigma}^{(a)} \circ F q_{B},
\end{aligned}
\end{aligned}
$$

and

$$
\begin{aligned}
F h_{t}^{A} \circ \sigma^{(a)} & =F h_{t}^{A} \circ Q(a)_{D} \circ F \sigma \\
& =Q(a)_{A} \circ F h_{t}^{A} \circ F \sigma \\
& =Q(a)_{A} \circ F\left(h_{t}^{A} \circ \sigma\right) \\
& =Q(a)_{A} \circ F\left(\sigma \circ h_{t}\right)=\sigma^{(a)} \circ F h_{t},
\end{aligned}
$$

for all $t \in \mathbb{R}$. 
Remark 3.3. Given a fibered chart $\varphi:\left(q_{B}^{D}\right)^{-1}(\Omega) \rightarrow w(\Omega) \times \mathbb{R}^{n} \times \mathbb{R}^{q}$ of $D \rightarrow B$ and the local frame $\left\{\varepsilon_{j}, \varepsilon_{k}^{\prime}, 1 \leq j \leq n\right.$ and $\left.1 \leq k \leq q\right\}$ on $\Omega$ defined by

$$
\left\{\begin{array}{l}
\varepsilon_{j}(b)=\varphi^{-1}\left(w(b), e_{j}, 0\right), \\
\varepsilon_{k}^{\prime}(b)=\varphi^{-1}\left(w(b), 0, e_{k}^{\prime}\right),
\end{array}\right.
$$

where $\left(e_{j}\right),\left(e_{k}^{\prime}\right)$ are basis of $\mathbb{R}^{n}, \mathbb{R}^{q}$, respectively, let us denote $\left\{\varepsilon_{j \beta}\right.$, $\varepsilon_{k \beta}^{\prime}, 1 \leq j \leq n, 1 \leq k \leq q$ and the local frame associated the fibered chart $F \varphi$. We have

$$
\varepsilon_{j \beta}=\varepsilon_{j}^{\left(e_{\beta}\right)} \text { and } \varepsilon_{k \beta}^{\prime *}=\varepsilon_{k}^{\prime\left(e_{\beta}\right)}
$$

Indeed, let $c_{j}: \Omega \rightarrow \mathbb{R}^{n}, b \mapsto e_{j}$ and $c_{0}^{\prime}: \Omega \rightarrow \mathbb{R}^{q}, b \mapsto 0 ; F c_{j}$ and $F c_{0}^{\prime}$ are constant maps $\tilde{b} \mapsto e_{j 0}$, and $\tilde{b} \mapsto 0_{\left(V^{F}\right)^{q}}$ :

$$
\begin{aligned}
\varepsilon_{j}^{\left(e_{\alpha}\right)}(\tilde{b}) & =Q\left(e_{\alpha}\right)_{p^{-1}(U)} \circ F \varepsilon_{j}(\tilde{b}) \\
& =Q\left(e_{\alpha}\right)_{p^{-1}(U)} \circ F\left(\varphi^{-1} \circ\left(\omega \times c_{j} \times c_{0}^{\prime}\right)\right)(\widetilde{b}) \\
& =F \varphi^{-1} \circ Q\left(e_{\alpha}\right)_{u(U) \times \mathbb{R}^{n}}\left(F w(\tilde{b}), e_{j 0}, 0\right) \\
& =F \varphi^{-1}\left(F w(\widetilde{b}), e_{\alpha}, e_{j 0}, 0\right)=F \varphi^{-1}\left(F w(\tilde{b}), e_{j \alpha}, 0\right)=\varepsilon_{j \alpha}(\tilde{x})
\end{aligned}
$$

for $\tilde{b} \in F W$.

Corollary 3.3. Sections $\sigma^{(a)}, \sigma \in \Gamma\left(q_{B}^{D}\right)$ and $a \in A^{F}$, generate the module $\Gamma(F D)$ of smooth sections of the vector bundle (FD, FB, $\left.F\left(q_{B}^{D}\right)\right)$. 


\section{References}

[1] D. J. Eck, Product-preserving functors on smooth manifolds, Journal of Pure and Applied Algebra 42(2) (1986), 133-140.

DOI: https://doi.org/10.1016/0022-4049(86)90076-9

[2] J. Gancarzewicz, W. Mikulski and Z. Pogoda, Lifts of some tensor fields and connections to product preserving functors, Nagoya Mathematical Journal 135 (1994), 1-41.

DOI: https://doi.org/10.1017/S0027763000004931

[3] J. Grabowski and M. Rotkiewicz, Higher vector bundles and multi-graded symplectic manifolds, Journal of Geometry and Physics 59(9) (2009), 1285-1305.

DOI: https://doi.org/10.1016/j.geomphys.2009.06.009

[4] J. Grabowski and M. Rotkiewicz, Graded bundles and homogeneity structures, Journal of Geometry and Physics 62(1) (2012), 21-36.

DOI: https://doi.org/10.1016/j.geomphys.2011.09.004

[5] G. Kainz and P. W. Michor, Natural transformations in differential geometry, Czechoslovak Mathematical Journal 37(4) (1987), 584-607.

[6] I. Kolář, Covariant approach to natural transformations of Weil bundles, Commentationes Mathematicae Universitatis Carolinae 27(4) (1986), 723-729.

[7] I. Koláŕ, On the natural operators on vector fields, Annals of Global Analysis and Geometry 6(2) (1988), 119-117.

\section{DOI: https://doi.org/10.1007/BF00133034}

[8] I. Kolář and M. Modugno, Torsions of connections on some natural bundles, Differential Geometry and its Applications 2(1) (1992), 1-16.

DOI: https://doi.org/10.1016/0926-2245(92)90006-9

[9] I. Koláŕ, J. Slovák and P. W. Michor, Natural Operations in Differential Geometry, Springer-Verlag, Berlin, Heidelberg, 1993.

DOI: https://doi.org/10.1007/978-3-662-02950-3

[10] O. O. Luciano, Categories of Multiplicative Functors and Morimoto's Conjecture, Preprint No. 46, Institut Foudrier, Laboratoire de Mathématiques, Grenoble, 1986.

[11] K. C. H. Mackenzie, General Theory of Lie Groupoids and Lie Algebroids, Lecture Notes Series, no. 213, London Math. Soc., Cambridge Univ. Press, 2005.

[12] W. M. Mikulski, Product preserving gauge bundle functors on vector bundles, Colloquium Mathematicum 90(2) (2001), 277-285.

DOI: https://doi.org/10.4064/cm90-2-7

[13] W. M. Mikulski and M. Kures, Liftings of linear vector fields to product preserving gauge bundle functors on vector bundles, Lobachevskii Journal of Mathematics 12 (2003), 51-61. 
[14] A. Ntyam, G. F. Wankap Nono and Bitjong Ndombol, On lifts of some projectable vector fields associated to a product preserving gauge bundle functor on vector bundles, Archivum Mathematicum 50(3) (2014), 161-169.

DOI: https://doi.org/10.5817/AM2014-3-161

[15] A. Ntyam, G. F. Wankap Nono and Bitjong Ndombol, Some further results on lifts of linear vector fields related to product preserving gauge bundle functors on vector bundles, Archivum Mathematicum 52(3) (2016), 131-140.

DOI: https://doi.org/10.5817/AM2016-3-131

[16] A. Weil, Théorie des Points Proches sur les Variétés Différentiables, Topologie et Géométrie Différentielle, Colloque du CNRS, Strasbourg, 1953 (CNRS, Paris, 1953), 97-110. 